\title{
Announcement: Howard Rosenbrock Prize 2015
}

\author{
Miguel F. Anjos 1
}

Published online: 1 September 2016

(c) Springer Science+Business Media New York 2016

It is my great pleasure to announce that the recipients of the Howard Rosenbrock Prize 2015 are

\section{Moritz Simon and Michael Ulbrich}

of the Department of Mathematics, Technische Universität München.

Their winning article is entitled

Adjoint based optimal control of partially miscible two-phase flow in porous media with applications to $\mathrm{CO}_{2}$ sequestration in underground reservoirs (Simon and Ulbrich 2015).

The citation reads:

This article is an excellent example of advances in applied mathematics that address important technological challenges.

The study develops optimal control strategies for $\mathrm{CO}_{2}$ well injection to underground reservoirs through the synthesis of the high fidelity of two-phase flow in porous media, state of the art PDE-constrained optimization formulations and modeling environments, and advanced optimization strategies.

The results demonstrate how to maximize the sequestration of $\mathrm{CO}_{2}$, and the work is an excellent contribution to the development of sustainable strategies for the management of $\mathrm{CO}_{2}$ for the environment.

The Howard Rosenbrock Prize is sponsored by Springer, and the winners receive a cash award of USD \$500 and individual plaques.

Miguel F. Anjos

anjos@stanfordalumni.org

1 GERAD \& Polytechnique Montréal, C.P. 6079, Succ. Centre-ville, Montreal, QC H3C 3A7, Canada 
Moritz Simon (left) and Michael Ulbrich. (Photo credit: Sebastian Garreis)

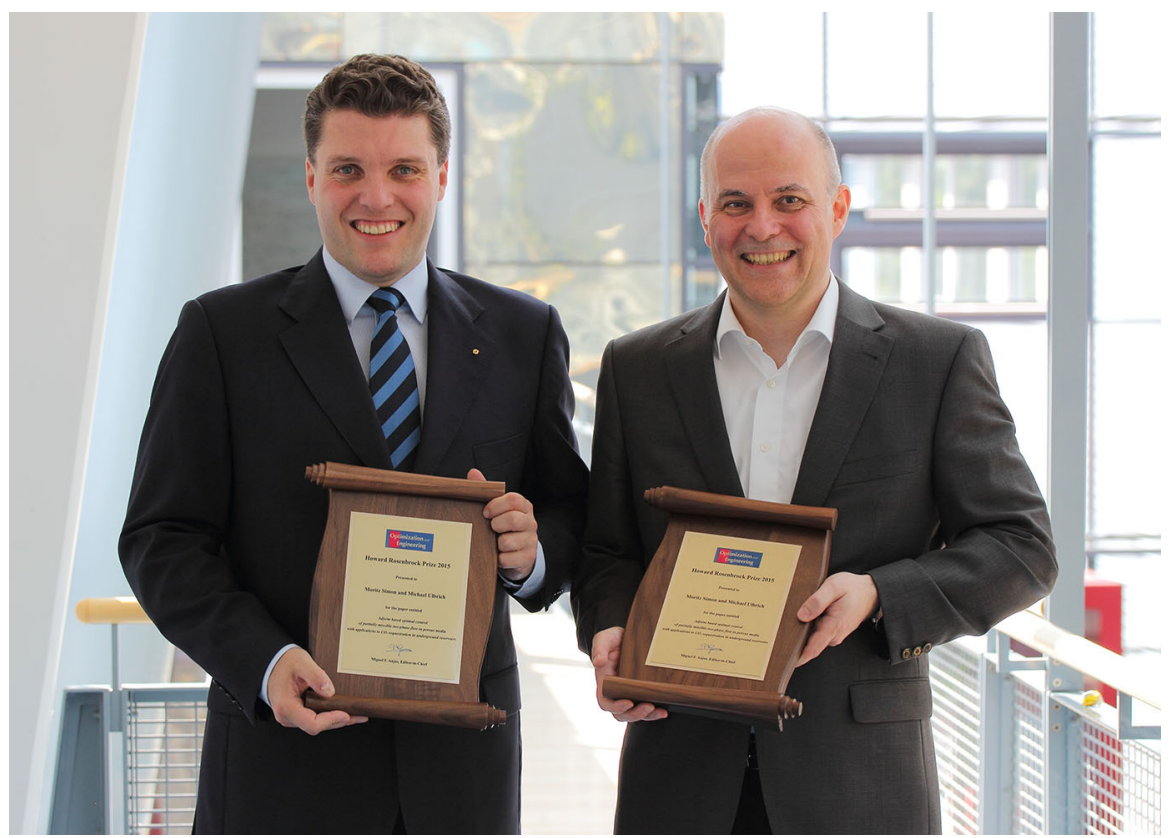

OPTE is a multidisciplinary journal that provides a forum where engineering researchers can obtain information about new developments in optimization, and optimization researchers can learn about the successes of optimization in engineering fields. The prize is named after Howard Rosenbrock, who embodied this bridging of the gap between optimization and engineering (Anjos 2015).

The winning paper was selected by a committee of OPTE editors, chaired by Joaquim R. R. A. Martins, and composed of Shabbir Ahmed, Lorenz Biegler, Roy H. Kwon, and Stein W. Wallace. I am grateful to them for contributing their time and expertise to the selection process.

I encourage all readers of OPTE to submit their high-quality papers on any aspect of the interface of optimization and engineering. You may be a future recipient of the Howard Rosenbrock Prize!

\section{References}

Anjos MF (2015) Announcement: Inaugural Howard Rosenbrock Prize. Optim Eng 16(3):507-509

Simon M, Ulbrich M (2015) Adjoint based optimal control of partially miscible two-phase flow in porous media with applications to $\mathrm{CO}_{2}$ sequestration in underground reservoirs. Optim Eng 16(1):103-130 